\title{
X-Ray Sources With No Counterparts in OTher Wavelength BandS
}

\author{
Chulhee Kim ${ }^{1}$, Byung-Kwon Moon ${ }^{1}$, And Hong Soo PARK ${ }^{2}$ \\ ${ }^{1}$ Division of Science Education and Institute of Fusion Science, Chonbuk National University, Jeonju 561-756, Korea \\ ckim@chonbuk.ac.kr, moonbk@jbnu.ac.kr \\ ${ }^{2}$ Korea Astronomy and Space Science Institute, Daejeon 305-348, Korea; hspark@kasi.re.kr
}

Received June 30, 2014; accepted September 18, 2014

\begin{abstract}
In order to identify peculiar X-ray sources, we select 442 sources with no counterparts in other wavelength bands (as of the year 1999) from the ROSAT All-Sky Survey Bright Source Catalog. We crosscorrelate this initial list with the NASA/IPAC Extragalactic Database, the USNO and WISE catalogs, and the HEASARC XRAY Master Catalog. Eventually, we are left with four unidentified sources with no counterparts in other wavelength bands. We present these four sources and their X-ray properties in this paper.
\end{abstract}

Key words: X-rays: general — Methods: data analysis — Astronomical data bases: miscellaneous

\section{INTRODUCTION}

Astronomy has made great strides by discovering new types of previously unknown celestial objects. Many investigations and studies have solely concentrated on well-known objects. Among the various all-sky survey projects, numerous objects have been detected, but only well-known objects have remained the target of continuous investigation. Unidentified objects have been ignored despite their potential for use. If new undiscovered types of object exist, they may well hide behind the identified objects. It is therefore necessary to pay attention to unidentified objects in order to find possible new types of object. For the identification of new objects, the survey data in the high-energy range will be a good starting point. Therefore, we analyze $\mathrm{X}$-ray survey data in the present work.

The ROSAT All-Sky Survey (RASS) was conducted from June 1990 to February 1991. As the first all-sky survey performed with an imaging X-ray telescope, it provided a new opportunity for finding X-ray sources. In total, 18806 sources were cataloged down to a limiting ROSAT PSPC count-rate of $0.05 \mathrm{cts} / \mathrm{s}$ in the $0.1-2.4$ $\mathrm{keV}$ energy band, with a detection likelihood of at least 15 and with at least 15 source photons. The exposure time varied between about $400 \mathrm{~s}$ and $40000 \mathrm{~s}$ in the ecliptic plane and poles, respectively.

At a brightness limit of $0.1 \mathrm{cts} / \mathrm{s}$, the catalog achieves a sky coverage of $92 \%$. The typical position accuracy was $30^{\prime \prime}$. Voges et al. (1999, hereafter V1999) crosscorrelated the ROSAT All-Sky Survey Bright Source Catalog (RBSC) with various catalogs, including public databases like the NASA/IPAC Extragalactic Database (NED) or SIMBAD, the latest versions of catalogs published or in press, as well as lists that were made available to them via private communications (see Table 3 in their paper for the list of all catalogs). Since the publication of V1999, the RASS database has been used

Corresponding author: B.-K. Moon for the search for optical (Wang et al. 2014; Parejko et al. 2008; Zickgraf et al. 2003) and radio (Anderson \& Filipovic 2009) counterparts of X-ray sources.

Our study focuses on the large number of neglected objects observed in these survey projects. Our approach bears the potential of discovering new types of $\mathrm{X}$-ray sources very different from well-known sources such as, e.g., AGN and pulsars.

\section{DATA AND ANALYSIS}

In the RBSC, we selected 442 sources with no counterparts in other wavelength bands within a search radius of $5^{\prime}$. These sources were identified via comparison with 15 existing databases including SIMBAD, NED, and VERON (see Table 3 in V1999) for both stellar and extended objects. Given that this selection is based on results obtained 15 years ago (by V1999), we checked the identification of these 442 sources by searching the NED, leaving us with 155 sources without non-X-ray counterparts. The search radius was $30^{\prime \prime}$, which is the maximum permitted value in NED.

The RBSC sources were correlated with the TYCHO catalog (Hog et al. 1998) in order to assess their positional accuracies. As TYCHO contains only stars, this comparison gives the positional accuracies of point-like sources. Figure 8 in V1999 shows a correlation of the RBSC with the TYCHO catalog entries for a search radius up to $120^{\prime \prime}$. The comparison shows that $90 \%$ of the RBSC sources are found within $25^{\prime \prime}$ of the optical position. Therefore, a search radius of $30^{\prime \prime}$ is reasonable for the identification of our 155 sources.

In addition, the United States Naval Observatory USNO-B1.0 and Wide Field Infrared Survey Explorer (WISE) (Wright et al. 2010) catalogs were released recently but have not yet been incorporated into NED. Hence, we searched these two catalogs separately for possible counterparts of our 155 X-ray sources.

The USNO-B catalog is a compilation of optical astrometric and photometric measurements for more than 
1RXS J130236.8-285654

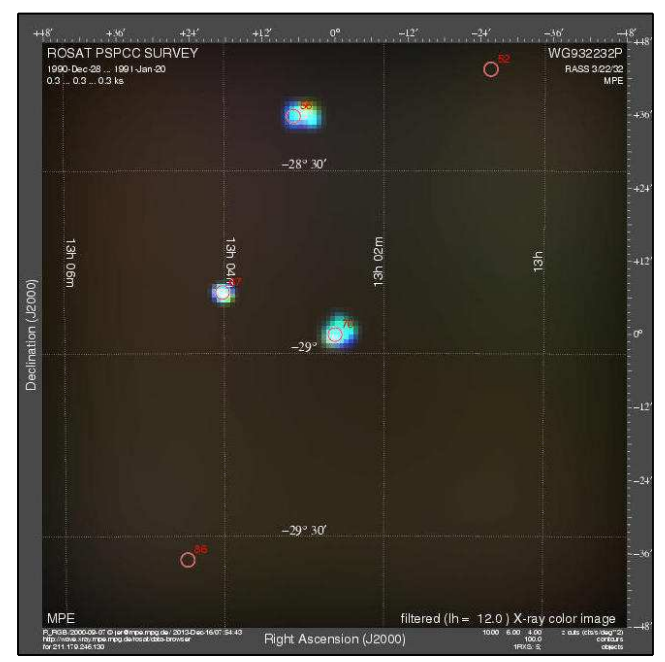

1RXS J074306.1-642820

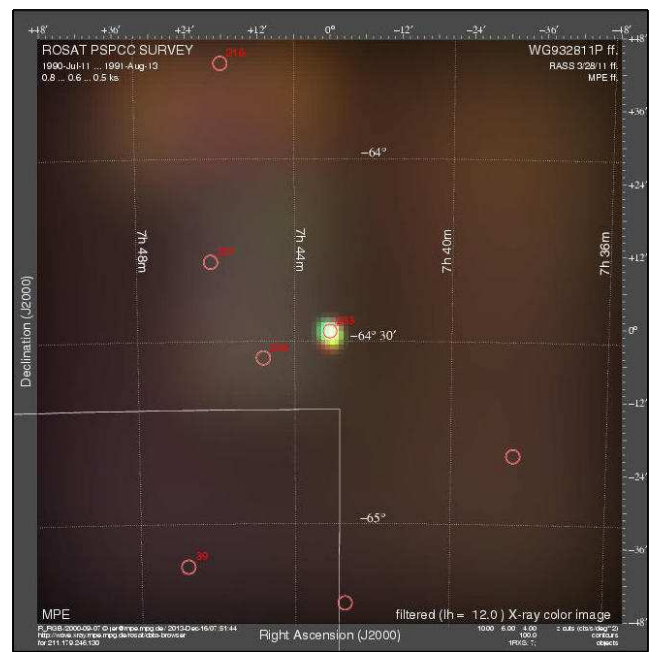

1RXS J175811.1-405516

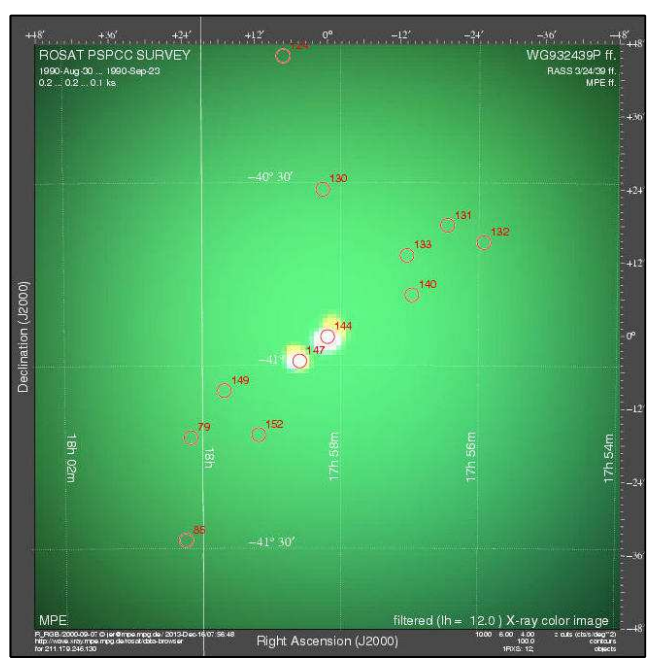

1RXS J222933.8-431952

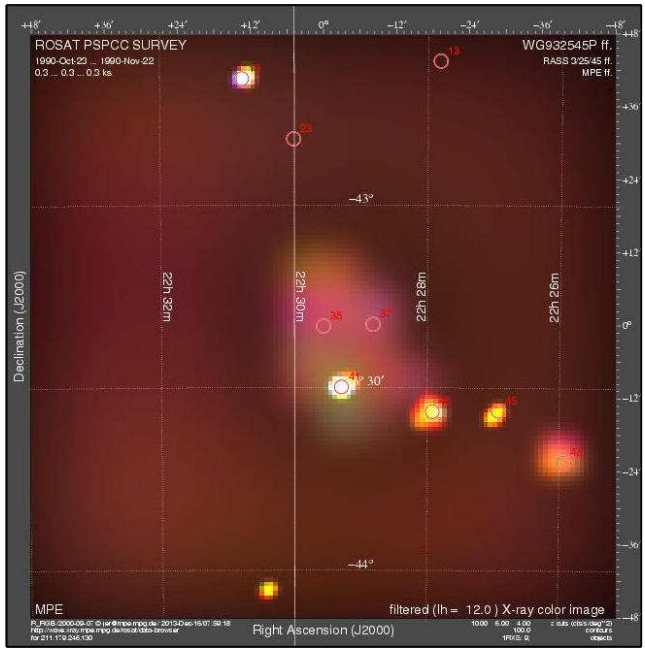

Figure 1. Soft $\mathrm{X}$-ray images of our four sources without counterparts at other wavelengths. In each figure, the source is located at the center. X-ray color images were produced by mapping three selected energy bands to red, green, and blue, respectively. Red circles with numbers indicate the positions of sources in the catalog list. For details see the description of the ROSAT Data Browser at www.xray.mpe.mpg.de/cgi-bin/rosat/data-browser/x/ .

one billion objects over the entire sky (Monet et al. 2003). The catalog was generated from digital scans of 7435 Schmidt plates collected in various sky surveys during the last 50 years. USNO-B1.0 is supposed to provide full-sky coverage, completeness down to apparent optical magnitude $\mathrm{V}=21$, a mean astrometric accuracy of $0.2^{\prime \prime}, 0.3$ magnitude photometric accuracy in up to five filters, and a success rate of $85 \%$ for distinguishing stars from non-stellar objects.

WISE performed an all-sky infrared survey at 3.4, 4.6, 12 , and $22 \mu \mathrm{m}$ using a $40-\mathrm{cm}$ aperture infrared space telescope. The survey aimed at a sky coverage of $99 \%$, with at least eight images taken of each sky position. The spatial resolution achieved in the four bands is $6.1^{\prime \prime}, 6.4^{\prime \prime}, 6.5^{\prime \prime}$, and $12.0^{\prime \prime}$, respectively. WISE achieved $5 \sigma$ point source sensitivities of $0.08,0.11,1$, and $6 \mathrm{mJy}$, respectively, in unconfused regions on the ecliptic. The WISE Source Catalog in the WISE AllSky Data Release contains information on 563921584 objects.

Using the WISE catalog, we found 32 sources (out of 155 initially) with no counterpart in other wavelength bands within a search radius of $12^{\prime \prime}$. (12" is the maximum position accuracy of WISE.) Of these 32, we eventually identified four objects without counterparts in USNO-B within a search radius of $5^{\prime \prime}$. Although $3^{\prime \prime}$ is the maximum position uncertainty of USNO-B, no source could be identified for search radii of $3^{\prime \prime}$ and $4^{\prime \prime}$. As a further check, we tried to identify our four sources in the HEASARC XRAY Master Catalog which combines the results from X-ray observatories like Chandra, Swift, or XMM-Newton. We also searched the Sloan 
Table 1

Physical parameters of our four peculiar X-ray sources

\begin{tabular}{cccccccccccc}
\hline 1RXS source name & $R A$ & $D E C$ & $n_{H}^{\mathrm{a}}$ & $\mathrm{HR}^{\mathrm{b}}$ & $\mathrm{HR}^{\mathrm{c}}$ & ext $^{\mathrm{d}}$ & ext1 $^{\mathrm{e}}$ & $\exp ^{\mathrm{f}}$ & cps $^{\mathrm{g}}$ & flux $^{\mathrm{h}}$ & flux $^{\mathrm{i}}$ \\
\hline J074306.1-642820 & 115.7754 & -64.4724 & 1.245 & 0.15 & -0.37 & 19 & 1 & 509 & 0.051 & 22.96 & 4.625 \\
J130236.8-285654 & 195.6533 & -28.9483 & 0.649 & 0.84 & -0.10 & 41 & 3 & 290 & 0.065 & 22.08 & 8.359 \\
J175811.1-405516 & 269.5462 & -40.9211 & 1.458 & -0.27 & -0.36 & 22 & 2 & 170 & 0.113 & 54.94 & 7.801 \\
J222933.8-431952 & 337.3908 & -43.3312 & 0.176 & 0.30 & -0.24 & 0 & 0 & 307 & 0.056 & 8.293 & 5.584 \\
\hline
\end{tabular}

a galactic HI column density in units of $10^{21} \mathrm{H}$-atoms $/ \mathrm{cm}^{2}$

b hardness ratio 1

c hardness ratio 2

d source extent in arcsec

e likelihood of source extent

$\mathrm{f}$ exposure time in sec

$\mathrm{g}$ source count rate (vignetting corrected) in counts/sec

$\mathrm{h}$ flux in the $0.1-2.4 \mathrm{keV}$ energy range in units of $10^{-13} \mathrm{erg} \mathrm{cm}^{-2} \mathrm{~s}^{-1}$, assuming a power law (useful for AGN, clusters of galaxies)

i flux in units of $10^{-13} \mathrm{erg} \mathrm{cm}^{-2} \mathrm{~s}^{-1}$, with empirical conversion between count-rates and fluxes following Schmidt \& Green (1986)
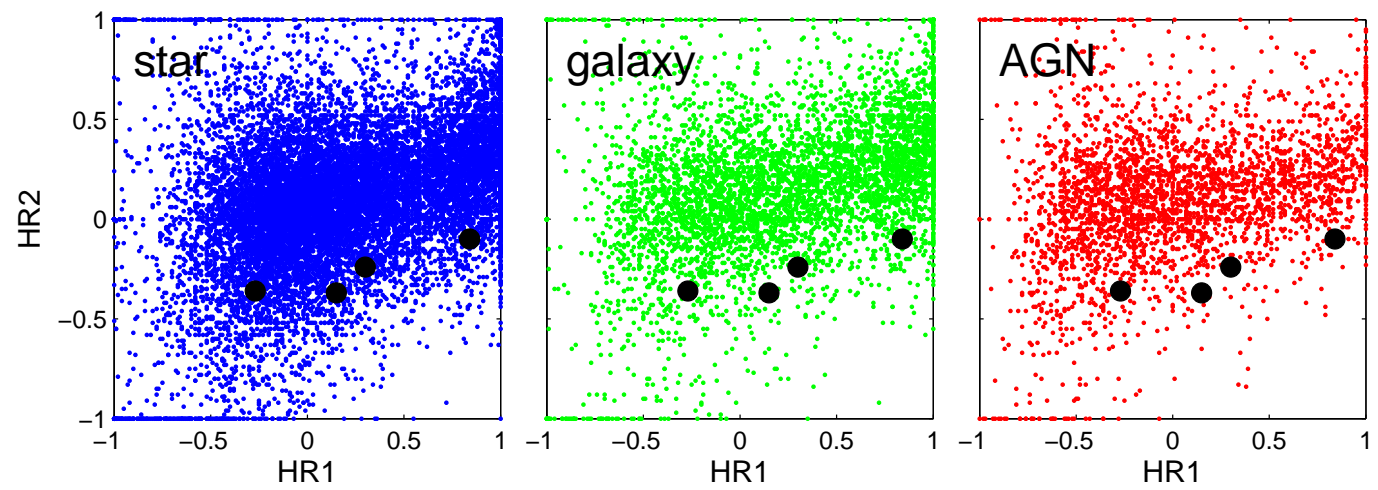

Figure 2. Our four sources (black points) in the [HR1]:[HR2] plane, together with the distributions for stars (left), galaxies (center), and AGN (right).

Digital Sky Survey (SDSS) Data Release 8 database but did not find our four sources there. Accordingly, our four sources can be identified only as X-ray sources with no known counterparts in any other wavelength band. Figure 1 shows X-ray images of the four sources, Table 1 shows their key parameters.

The angular size of 1RXS J222933.8-431952 is listed as zero in Table 1, and a distinct diffuse cloud can be seen around the source. Indeed, the detection of this source could be spurious because the diffuse X-ray cloud can interfere with position or flux measurements.

We compared the X-ray properties of our four sources with those of stars, galaxies, and active galactic nuclei (AGN) in order to find clues to their classification. To achieve this, we investigated the correlations between all possible pairs of the parameters in Table 1. As an example, Figure 2 shows various distributions of the spectral hardness ratios (X-ray colors) HR1 and HR2. The three panels show the hardness ratio distributions for stars, galaxies, and AGN, respectively, together with our four sources. Because the distributions of stars, galaxies, and AGN in the [HR1]:[HR2] plane are quite similar, we cannot distinguish which object type among stars, galaxies, and AGN might agree best with our four sources. We obtained similar results for all other pairs of parameters.

\section{Conclusions}

We have identified four X-ray sources with no counterparts in other wavelength bands. The identification of 1RXS J222933.8-431952 is potentially spurious. In the image of 1RXS J222933.8-431952 one finds an extended cloud-like object, a background source whose size is greater than $2^{\prime}$. However, it is unclear whether this cloud is physically related to 1RXS J222933.8-431952.

The four sources of interest seem to be rather ordinary X-ray sources and have no peculiar and/or extreme characteristics in terms of their X-ray parameters. However, it is somewhat surprising that they emit only in X-rays with no UV, IR, optical, or radio emission. Therefore, further investigations of these four sources at other wavelengths, as well as additional $\mathrm{X}$-ray observations, are required. Indeed, we proposed dedicated X-ray observations but were not awarded the necessary observing time. Accordingly, we decided to report our results despite a lack of further observations in order to motivate further searches for unidentified sources, seeking new types of objects and/or new astronomical phenomena. 


\section{ACKNOWLedgments}

This research has made use of the NASA/IPAC Extragalactic Database (NED) which is operated by the Jet Propulsion Laboratory, California Institute of Technology, under contract with the National Aeronautics and Space Administration. This publication makes use of data products from the Wide-field Infrared Survey Explorer, which is a joint project of the University of California, Los Angeles, and the Jet Propulsion Laboratory/California Institute of Technology, funded by the National Aeronautics and Space Administration.

\section{REFERENCES}

Anderson, M. W. B., \& Filipovic, M. D. 2009, Radio Detection of 18 RASS BL Lac Objects, Serb. Astron. J., 179, 7

Hog, E., et al. 1998, The TYCHO Reference Catalogue, A\&A, 335, 65
Monet, D. G., et al. 2003, The USNO-B Catalog, AJ, 125, 984

Parejko, J. K., et al. 2008, Source Matching in the SDSS and RASS: Which Galaxies are Really X-Ray Sources?, AJ, 135, 10

Schmidt, M., \& Green, R. F. 1986, Counts, Evolution, and Background Contribution of X-Ray Quasars and Other Extragalactic X-Ray Sources, ApJ, 305, 68

Voges, W., et al. 1999, The ROSAT All-Sky Bright Source Catalogue, A\&A, 349, 389 (V1999)

Wang, L., et al. 2014, Measuring the X-Ray Luminosities of SDSS DR7 Clusters from ROSAT All Sky Survey, MNRAS, 439, 611

Wright, E. L., et al. 2010, The Wide-Field Infrared Survey Explorer (WISE): Mission Description and Initial OnOrbit Performance, AJ, 140, 1868

Zickgraf, F. J., et al. 2003, The Hamburg/RASS Catalogue of Optical Identifications. Northern High-Galactic Latitude ROSAT Bright Source Catalogue X-Ray Sources, A\&A, 406, 535 